\title{
ANÁLISIS MULTIDIMENSIONAL DEL APRENDIZAJE DE LENGUA EXTRANJERA EN ESPORTS
}

\author{
Ana Yara Postigo Fuentes \\ Universidad de Málaga (España) \\ anayaratranslator@gmail.com · https://orcid.org/0000-0001-7965-1911 \\ Manuel Fernández Navas \\ Universidad de Málaga (España) \\ mfernandez1@uma.es · https://orcid.org/0000-0002-9445-2643
}

\begin{abstract}
Resumen. El peso que ha adquirido la industria de los videojuegos en diferentes países y la posibilidad de jugar a estos videojuegos en línea han permitido que jugadores españoles se comuniquen con jugadores de otros orígenes que hablen una lengua diferente a la de ellos. En este estudio de caso, hemos querido comprender en profundidad el conocimiento de la lengua extranjera desde diferentes dimensiones, con el objetivo de conocer qué situaciones informales vive el alumnado en su vida diaria para potenciar el aprendizaje de la lengua extranjera. Para ello se han llevado a cabo observación sistemática y análisis de los discursos en partidas, entrevistas a jugadores y evaluación de destrezas. Los resultados obtenidos se han puesto en relación con la escala descriptiva que ofrece el Marco Común Europeo de Referencia de las Lenguas (MCERL).
\end{abstract}

Palabras clave: Lengua Extranjera, Aprendizaje de lenguas, Proceso de comunicación, Aprendizaje informal, eSport.

\section{A MULTIDIMENSIONAL ANALYIS OF LANGUAGE LEARNING IN ESPORTS}

\begin{abstract}
The importance that videogame industry has gained in different countries and the possibility of playing these videogames online have allowed Spanish players to communicate with players that speak a different language from theirs. Through this case study, we aimed to get a multidimensional understanding of the knowledge about a foreign language, in order to learn about the informal context that students daily live and improve their language learning. For that purpose, the used instruments to collect data were systematic observation and analysis of the oral output during gaming, interviews with players and assessment of skills. The results have been linked to the descriptive scale offered by the Common European Framework of References for Language (CEFR).
\end{abstract}

Keywords: Foreign Language, Languages learning, Communication process, Informal learning, eSport. 


\title{
ANÁLISE MULTIDIMENSIONAL DA APRENDIZAGEM DA LÍNGUA ESTRANGEIRA EM ESPORTS
}

\begin{abstract}
Resumo. O peso que tem adquirido a indústria dos videojogos em diferentes países e a possibilidade jogar estes videojogos online têm permitido que jogadores espanhóis se comuniquem com jogadores de outras origens que falem uma língua diferente à deles. Neste estudo de caso, pretendemos compreender em profundidade o conhecimento da língua estrangeira desde diferentes dimensões, com o objetivo de conhecer que situações informais vivenciam os alunos nas suas vidas quotidianas para potenciar a aprendizagem da língua estrangeira. Por isso, levaram-se a cabo observação sistemática e análise dos discursos em jogos, entrevistas a jogadores e avaliação das suas habilidades. Os resultados obtidos compararam-se com a escala descritiva que oferece o Marco Comum Europeu de Referência das Línguas (MCERL)
\end{abstract}

Palavras-chave: Língua estrangeira, Aprendizagem de línguas, Processos de comunicação, Aprendizagem informal, eSport

\section{Introducción}

El peso que ha adquirido la industria de los videojuegos en diferentes países ha hecho que investigadores y académicos vuelquen la mirada en este ámbito. Este crecimiento se ha visto especialmente reflejado en los eSports, que podríamos definir como

«competiciones profesionales de videojuegos que se celebran cada dos semanas aproximadamente, y que miles de aficionados siguen a través de servicios como YouTube y Twitch. En estas retrasmisiones en directo les acompañan comentaristas profesionales que explican las complejidades de las diferentes estrategias de juego. Cada mes, se celebra un torneo más grande que reúne a equipos de todo el mundo y en el que los jugadores pueden disfrutar de la atmósfera en persona, con enormes pantallas que representan lo que está pasando en el juego» (Educo, 2017).

Como resultado de este auge, nace la Cátedra Estratégica de eSports en la Universidad de Málaga, en la que se encuadra esta investigación y se le agradece su incondicional apoyo y, en especial, a la Liga Survival, donde se lleva a cabo el trabajo de campo.

La globalización de la industria también ha forzado a estos jugadores a jugar en una lengua diferente a su lengua materna. Ambas situaciones llevan a los jugadores a entrar en contacto en una situación informal con una lengua extranjera. Quizás el hecho de que una gran parte de los videojuegos se desarrollen en inglés, que los comentaristas (o casters, como se les denomina en la jerga) describan las partidas en esta lengua o que los foros angloparlantes sean aquellos que actualizan antes las novedades que puedan aparecer en este campo han hecho del inglés la lengua franca en el mundo de los videojuegos.

Siendo docente de lengua extranjera sabemos que el primer contacto del alumnado con la lengua extranjera que queremos que aprenda no será en la clase, como solía suceder. Antes de comenzar a asistir a clase y paralelamente, el alumnado está expuesto a toda la información que pueda interesarle en el idioma de origen gracias a 
internet (Fernández, 2006). En este estilo de vida saturada de relaciones sociales virtuales y de interacciones más o menos lúdicas con la pantalla (Pérez Gómez, 2012), tenemos que volcar la mirada hacia el aprendizaje informal, que también puede ayudar a aprender en contextos complejos, inciertos, multidimensionales y que permite aprender indagando, flexibilidad que ofrece este nuevo entorno. Los espacios de aprendizaje informal, como es el caso de los entornos familiares o de las actividades de aprendizaje social menos regladas, se muestran más flexibles y próximos a las nuevas formas culturales de aprender (Pozo, 2016). Como docentes, no podemos pasar por alto esta situación y sacar el mayor provecho de ella. Así, muchos investigadores y académicos se han interesado por cómo se aprenden lenguas extranjeras cuando se juega a videojuegos (Chen, 2014; Chik, 2014; Gee, 2007; Godwin-Jones, 2014; Reinders, 2014; Reinhardt, y Sykes, 2014(a); 2014(b); Sundqvist, y Sylvén, 2012(a); 2012(b), 2015).

Nuestro objetivo en esta investigación es comprender los procesos de aprendizaje de una lengua extranjera en el entorno de los eSports. Para ello necesitábamos entender en profundidad cuál era el conocimiento de la lengua extranjera en todas sus dimensiones por parte de los jugadores. Con esta finalidad resultaba idóneo utilizar un instrumento aceptado de manera generalizada como es el Marco Común de Referencia de las Lenguas (MCERL). Este instrumento nos sirve para analizar los contenidos lingüísticos, puesto que actualmente el nivel de lengua extranjera se mide de acuerdo a este marco, en el que el conocimiento de la lengua pasa a tener un valor de cambio por la calificación de acuerdo al nivel que hayan alcanzado a través de las pruebas (Alcaraz, 2015; Pérez Gómez, 2000), pero también desde un punto de vista comunicativo, entendiendo al jugador/aprendiz como agente social y hablante intercultural (Centro Virtual Cervantes, 2018). Comprendiendo en profundidad el conocimiento de la lengua extranjera desde diferentes dimensiones, podremos conocer qué situaciones informales vive el alumnado en su vida diaria para potenciar el aprendizaje de la lengua extranjera a través de un diseño didáctico que aproveche estos aspectos

\section{Aprendizaje de lenguas con videojuegos}

Entre los valores educativos que podemos encontrar en los videojuegos encontramos la potenciación de la curiosidad por aprender, el favorecimiento de determinadas habilidades, el desarrollo de distintas áreas transversales y el refuerzo de la autoestima (Martín Díaz, 2012). Entre estas áreas transversales encontramos el trabajo de competencias como el pensamiento reflexivo y el razonamiento, la atención y la memoria, la habilidad óculo-manual, la habilidad para la resolución de conflictos, la identificación y aprendizaje de vocabulario y conceptos numéricos y la puesta en contacto del sujeto que juega con normas de socialización y qué comportamientos son aceptados en ellas (Pindado, 2005). Esto es coherente con el aprendizaje relevante que propone Pérez Gómez (2004) en el cual destaca la importancia de la motivación intrínseca y de la práctica para que se produzca un aprendizaje de calidad. Referencia también a esta relación entre curiosidad, intereses y motivación intrínseca hace Sampedro Requena (2012) al relacionarlo con el aprendizaje significativo.

Es necesario entender que existen principios para fomentar aprendizajes de gran calidad dentro de los videojuegos (Gee y Hayes, 2010; Whymaps, 2017), especialmente en los más modernos donde las posibilidades de toma de decisiones del jugador dentro de la narrativa del juego se han multiplicado de forma exponencial en relación con los videojuegos más antiguos. Entre estos principios es necesario destacar:

- La inmersión del jugador en una narrativa propia dónde el mismo puede tomar decisiones que afectan a la historia en función de sus intereses y conocimientos. 
- La asunción de roles diferentes en los diferentes contextos que ofrece la narrativa del juego.

- La posibilidad de realizar una práctica en el juego, en un entorno seguro en el que el jugador puede equivocarse tantas veces como quiera y aprender de sus errores.

- La adecuación de su práctica a las diferentes situaciones y contextos que ofrece la narrativa del juego.

- El enfoque de los videojuegos basado en la resolución de problemas con total libertad y con todos los medios a su alcance.

- El desarrollo de la metacognición a través de la propia evolución del jugador, de su avatar y del avance en la narrativa del juego.

- El desarrollo de las habilidades y conocimientos necesarios para completar con éxito la historia del juego -y que en muchas ocasiones obliga a recurrir a contenidos transversales y a conocimientos sobre diferentes disciplinas académicas como la historia, geografía, etc. (Begg, Dewhurst, y Macleod, 2005).

Estos principios de aprendizaje están íntimamente relacionados con las tres dimensiones del aprendizaje a través de la que el MCERL (2002) se desarrolla, ya que se tiene en cuenta al aprendiz como agente social, como hablante intercultural y como aprendiente autónomo. En este artículo nos centraremos en las dos primeras dimensiones señaladas.

Son muchas las teorías sobre cómo adquirimos una segunda lengua. Lo que es clave, tal y como ya exponía la hipótesis del input en el modelo del monitor de Krashen (1981), es que la adquisición de la lengua aumenta con lo que llama input comprensible, por lo que se deduce, por un lado, que la adquisición es la causa del desarrollo de las habilidades comunicativas, no lo contrario; y, por otro, los aprendices desarrollan la adquisición de gramática si el input está presente de manera abundante y comprensible. Así, el videojuego funciona en este caso como pretexto para propiciar diálogos, intercambios de opinión y conocimientos exponiendo al jugador a constante input.

\section{Método}

El trabajo de campo se ha llevado a cabo en una liga de eSport, es decir, de videojuegos que se juegan a nivel competitivo, en los torneos de los juegos League of Legends, Hearthstone y Clash Royale. Se ha escogido este contexto por ser una liga amateur, es decir, los jugadores no son profesionales.

La liga Survival tiene 5 meses de duración. Como peculiaridad con respecto a otras ligas, esta se juega de forma presencial cada viernes y sábado. Es decir, los equipos se enfrentan en un mismo espacio físico, en vez de hacerlo online. Las partidas son comentadas por casters y analistas que se encargan de transmitir la emoción de los enfrentamientos y explicar los matices de las jugadas.

Cabe destacar que todos los jugadores de esta liga son varones, por lo que el género no ha sido determinante en la selección del muestreo teórico. Se trata de personas que han percibido que existe una relación entre su uso de los videojuegos, tanto a nivel competitivo como a nivel de aficionado, con el aprendizaje de una lengua extranjera. Dentro de los jugadores que habían mostrado interés se han seleccionado de diferentes edades. La edad de los jugadores va entre los 18 y los 30 años. Ningún jugador es nativo o bilingüe de esta lengua extranjera. Todos juegan entre dos y doce 
horas diarias a videojuegos, de acuerdo con la información recogida en las entrevistas. No existe homogeneidad respecto a su ocupación: hay tanto estudiantes -bachillerato, formación profesional o estudios universitarios- como trabajadores en todos los sectores. Se trata de casos particularmente típicos (Flick, 2007) en lo que el objeto de la investigación se refiere. Con el objetivo de guardar el anonimato de todos los participantes, a los jugadores se nombrarán como J1, J2, etc., mientras que a la organización se nombrará como O1, O2, etc. Aunque muchos de ellos han manifestado abiertamente que no precisan el anonimato, se ha preferido mantener los preceptos éticos que forman parte de la investigación cualitativa.

Esta investigación es un estudio de caso, cuyo diseño se puede caracterizar de emergente, ya que se ha construido en interacción con el análisis de la información (Stake, 2010). La metodología empleada supone mi presencia como investigadora en el contexto en cuestión durante un periodo de tiempo suficiente para obtener la información necesaria para comprender en profundidad las claves de la práctica y pensamiento de los agentes. Las estrategias metodológicas en el trabajo de campo se han concretado en el diseño y desarrollo de la matriz reflejada en la Tabla 1, que incluye una amplia variedad de fuentes de información desde diferentes ángulos con la que se ha querido cumplir de forma sistemática con el requisito metodológico de la triangulación de datos (Gibbs, 2012; Santos Guerra, 2005; Simons, 2011) y explorar diferentes perspectivas y su interacción en contextos determinados y distintos.

Tabla 1

Fuentes de información y análisis de los datos

\begin{tabular}{|c|c|}
\hline Fuente de información & Análisis de los datos \\
\hline $\begin{array}{l}29 \text { entrevistas en grupo e individuales a } \\
\text { jugadores y organización }\end{array}$ & $\begin{array}{l}\text { Transcripción de las entrevistas y } \\
\text { categorización. Se ha hecho un análisis tras cada } \\
\text { entrevista para explorar la información necesaria }\end{array}$ \\
\hline 3 observaciones no participantes de partidas & $\begin{array}{c}\text { Se anotaron en el diario de investigación las } \\
\text { características de la comunicación en los e-sports } \\
\text { para profundizar en ellas a través de las otras } \\
\text { fuentes. }\end{array}$ \\
\hline $\begin{array}{l}\text { Análisis documental de } 8 \text { audios de los } \\
\text { jugadores en partidas }\end{array}$ & $\begin{array}{c}\text { Análisis de las mecánicas de comunicación } \\
\text { de los jugadores en las partidas y análisis del } \\
\text { léxico propio del juego }\end{array}$ \\
\hline $\begin{array}{l}\text { Análisis documental de vídeos de } 2 \text { partidas } \\
\text { comentadas }\end{array}$ & $\begin{array}{l}\text { Análisis de la prosodia del comentarista y } \\
\text { del léxico y mecánica propios del juego }\end{array}$ \\
\hline Pruebas de destrezas oral y escrita & $\begin{array}{l}\text { Se utilizan estos resultados como referencia } \\
\text { para saber qué nivel tienen con respecto a una } \\
\text { referencia de aceptación social }\end{array}$ \\
\hline
\end{tabular}

Se han realizado entrevistas tanto individuales como grupales a veintiocho jugadores. Con el mismo universo de intereses se ha realizado una entrevista grupal a dos personas parte del equipo organizador de la liga. Todas las entrevistas grabadas y transcritas han sido entrevistas formales que han cumplido rigurosamente el acuerdo ético del que se había informado antes a los participantes. Se dejaron de hacer entrevistas cuando se agotó la información. Antes de las entrevistas formales y la observación, se han mantenido un número indeterminado de conversaciones informales, tanto con la organización como con los jugadores, cuyo contenido a veces ha sido utilizado para la redacción de este documento. Estas conversaciones informales no se grabaron, pero las conclusiones se anotaron en el Diario de Investigación y han sido 
determinantes para la configuración del universo de intereses y la elaboración de las preguntas de investigación concretas para el diseño inicial de investigación acerca de la comunicación y que se detallan a continuación:

- ¿Cuánta comunicación en esta segunda lengua hay entre los jugadores?

- ¿Cómo es esa comunicación?

- ¿Es fluida?

- ¿Qué tipo de vocabulario usan?

- ¿Es una comunicación correcta o utilitaria?

- ¿Cómo es la negociación de significados?

- ¿Y la corrección de fallos?

Es necesario aclarar aquí que, en coherencia con el enfoque de investigación, estas preguntas no podían plantearse como hipótesis a confirmar en el trabajo de campo, sino como una concreción del foco inicial que ayuda a los investigadores a focalizar el diseño de investigación y a cómo conducirlo (Maxwell, 2010; Miles y Huberman, 1994) cuya finalidad última es comprender en profundidad el conocimiento de la lengua extranjera a través de los eSports. Todas las entrevistas formales fueron grabadas en audio digital y se desarrolló un análisis de datos entre una y otra, por lo que ha tenido lugar de manera simultánea a la recogida de información en el que el propio análisis reorientaba la investigación. Con este propósito, las entrevistas se transcribieron.

Se han seleccionado para la transcripción y la interpretación de la entrevista aquellas a quienes define Morse (1998) como «buenos informantes», es decir, jugadores que tienen a su disposición el conocimiento y la experiencia necesarios para responder a las preguntas en la entrevista, además de la capacidad para reflexionar sobre ello.

Antes de comenzar con las entrevistas formales y tras las entrevistas informales con diferentes jugadores y parte de la organización, se realizaron observaciones sistemáticas como no participante a dos partidas de League of Legends de dos jugadores en servidores europeos, donde podían estar expuestos a hablantes de otras lenguas diversas al español. La observación de la comunicación en las partidas se hace a través del chat escrito y de las conversaciones por voz a través de Teamspeak. Se trata de observaciones de tipo ecológico, tanto generales para ayudar a la elaboración del universo de intereses, como focalizadas para observar cuestiones concretas del uso de la segunda lengua que pudieran ser útiles para concretar el posterior análisis documental (Flick, 2007; Santos Guerra, 2005). Todas ellas se registraron en el Diario de Investigación.

El análisis documental se llevó a cabo en los audios de las partidas en semifinal y final con conversación entre los jugadores y en los vídeos de las partidas en semifinal comentados por los casters. Con el objetivo de contextualizar en el MCERL esta observación realizamos un análisis del discurso atendiendo a su contenido gramatical, funcional, léxico y pragmático, de manera que podamos entender qué nivel según qué contenidos se ponen en práctica cuando se juega una partida a este videojuego. Ejemplos obtenidos de este análisis ayudan a ilustrar los resultados en su apartado. Analizar otros factores del discurso también atendidos en el MCERL como los recursos de inteligibilidad o la dimensión cultural no es posible dentro de este análisis, puesto que no se manifiestan en estos documentos. Para completar esta información se han realizado las pruebas individuales a cada jugador e indagado en estos aspectos durante las entrevistas a través de preguntas sobre conversaciones con hablantes de otros países que reflejaran su realidad cultural y sobre los recursos de comunicación utilizados en conversaciones con estos hablantes. Las respuestas obtenidas se comparan con los 
contenidos adecuados a cada nivel (Centro Virtual Cervantes, 2018) para encuadrar los resultados. Esta nueva información nos ha servido para analizar esta realidad y sus procesos a través de la en la triangulación de información (Flick, 2007; Maxwell, 2012; Santos Guerra, 2005)

La prueba de inglés que se pasó a los jugadores determinaba sus niveles de destrezas de expresión y comprensión oral y escrito. La prueba oral consistía en llevar a cabo un fragmento de la entrevista en inglés, en la que los jugadores tenían que hablar sobre sus hábitos diarios en cuanto a videojuegos. La prueba escrita es una prueba estandarizada tipo test que se encuentra en la página web de los exámenes oficiales de la Universidad de Cambridge para conocer el nivel de inglés del participante según el Marco Común Europeo de Referencia de las Lenguas, Marco que utiliza esta institución para establecer sus niveles de exámenes. Este examen no es preceptivo y su objetivo es orientar al participante sobre el nivel del que parte. Para que haya correlación entre los exámenes escritos y orales, se propone también dividir los resultados de las pruebas orales entre los niveles del MCERL.

El análisis de toda esta información se hizo mediante un proceso de categorización (Flick, 2007; Maxwell, 2012; Santos Guerra, 2005) por el cual se transcribió toda la información para ser posteriormente agrupadas por temas o categorías, de forma que permitiera la aparición de categorías emergentes. Este proceso facilita la triangulación de información, así como el proceso de análisis de la misma cumpliendo con los criterios de validez en investigación cualitativa a los que se ha hecho mención con anterioridad.

\section{Resultados y discusión}

Dado que nos encontramos ante un trabajo de investigación que usa metodología de estudio de caso, los autores hemos decidido unir los apartados de resultados y discusión. Esto es así ya que según Flyvbjerg (2006) la metodología de estudio de caso ofrece amplias posibilidades para generar un conocimiento situado:

In the study of human affairs, there appears to exist only context-dependent knowledge, which, thus, presently rules out the possibility of epistemic theoretical construction (Flyvbjerg, 2006, p. 221).

En este sentido, no existen resultados «puros», por así decirlo, si no que estos siempre son dependientes del contexto y relacionados con él. Es por esto por lo que se han unido ambos apartados $\mathrm{y}$, aunque de alguna forma se ha intentado exponer resultados a parte de la discusión, eso no ha sido posible totalmente ya que hubiéramos sacrificado parte de la comprensión global del estudio.

\section{Resultados del análisis del discurso oral en interacción}

En esta modalidad de juego se les asigna de manera automática otros tres jugadores hasta completar el equipo. En las partidas se les une hablantes de otras lenguas, por lo que la lengua común pasa a ser automáticamente inglés para poder entenderse en el equipo. Así, la comunicación se produce por una doble vía: como el videojuego no permite el sistema de voz, los jugadores que se conocen entre sí se 
comunican por voz a través de Teamspeak. Con el resto de los jugadores lo harán a través del chat.

La comunicación de los jugadores entre sí se efectúa en español. Sin embargo, desde el principio, se incluyen abreviaturas típicas del juego, como GL (Good Luck), al principio del juego para desear buena suerte; WTF (What The Fuck), vulgarismo para expresar sorpresa en cualquier momento del juego o GG (Good Game), al final de la partida. Estas siglas las expresan en español, a pesar de referirse a términos en inglés. Otros términos que se utilizan en inglés a través del chat de voz son los tecnicismos del juego, como support, para referirse a uno de los roles del juego o top, para referirse a una de las líneas en las que sucede el juego. Además, algunos términos se adaptan al español, como pushear, del inglés push.

La comunicación en el chat se produce íntegramente en inglés. Se prima la rapidez y la efectividad de la comunicación en lugar de la corrección. Por ejemplo, al principio de la partida se definen los roles en el equipo. Uno de los jugadores pregunta: «u support?», en lugar de una frase más completa que sea correcta a nivel léxico o gramatical, como «are you going to play the role of support?». Aunque esta última forma es más correcta, desde un punto de vista comunicativo, la forma utilizada ha cumplido su cometido y el resto de los jugadores ha entendido el mensaje (Chen, 2014). A lo largo de la partida se pueden apreciar casos similares. Resulta también llamativa también la falta de uso de puntuación en el chat y que la separación de las frases u oraciones se haga mediante envío de estas al chat (Figueras Bates, 2014; Ridao Rodrigo y Rodríguez Muñoz, 2013). Tampoco se observan fórmulas de cortesía (Graham y Hardaker, 2017). Por ejemplo, si se quiere enviar a uno de los jugadores a apoyar en alguna de las líneas de batalla, se le dirá Go top, en lugar de incluir alguna fórmula de cortesía que implicaría un aumento de la longitud del discurso, como Would you mind going and supporting on the top line? A menudo se repiten las mismas peticiones si no se ha obtenido respuesta. Se hace uso de siglas, como sucedía en el chat de voz, y de términos técnicos propios del juego. Destacan el uso de vulgarismos en todos los diálogos (Chen, 2014). Durante ninguna de las dos partidas tiene lugar conversaciones a través de este chat sobre cualquier tema no relacionado con las tácticas del juego.

Atendiendo al contenido gramatical tanto oral como escrito, observamos que las oraciones no son largas o complejas, sino más bien sencillas y directas. Por ejemplo, no se formulan oraciones subordinadas, si no que se prefiere mantener una estructura simple. Son frecuentes las oraciones con infinitivo como I want to buy (utilizada cuando los jugadores vuelven a la base para adquirir elementos que puedan ser útiles en el desarrollo de la partida). Además, se utilizan formas abreviadas de orden en lugar de fórmulas de cortesía que impliquen el uso de verbos modales.

De acuerdo con el manual de introducción al MCERL para el inglés (EnglishProfile, 2011), estas estructuras serían típicas de A2, ya que para cuando el aprendiz alcanza este nivel, suele realizar oraciones de un máximo de 8 palabras de largo, aunque, como aclara Chen (2014), podría ser porque la inmediatez del juego así lo requiere. Además, se usan oraciones simples y no compuestas en su discurso, son capaces de usar oraciones descriptivas, utilizan con facilidad los pronombres interrogativos $w h$ - en preguntas y son capaces de usar diferentes verbos modales. En los niveles superiores encontramos un aumento de la complejidad en estos parámetros que no se aprecian en el contenido gramatical de nivel A2 que se observa en el discurso de la partida. Se atiende también al hecho de que una partida en la que la rapidez de la acción y la fluidez de comunicación son elementos fundamentales para la victoria, por lo que se tiende a la simplificación de las oraciones. 
El conocimiento de la lengua no se considera un fin en sí mismo, sino que se plantea un sentido funcional, esto es, qué se puede conseguir mediante el uso de la lengua, que podríamos relacionar con el valor de uso del conocimiento propio que propone la perspectiva constructivista del aprendizaje (Pérez, Soto, Sola y Serván, 2009). El Marco, de hecho, presenta una lista de funciones de la lengua propias de acuerdo con cada nivel (Centro Virtual Cervantes, 2018). Si hacemos un análisis del contenido funcional, es decir, qué consiguen comunicar los jugadores mediante el uso de la lengua extranjera, encontraremos que, usando estructuras simples, se describen aspectos de las partidas (como situación de otros jugadores o descripción de acciones), se hacen peticiones e invitaciones a acción y se expresa acuerdo o desacuerdo mediante sus intervenciones con los diálogos. Si bien este contenido podría ser de B1, dado que no existe complejidad en las estructuras, estaremos hablando aún de una representación de contenidos funcionales típicos de A2.

Con respecto al léxico, ambos jugadores se limitaron a hablar con el resto de los jugadores sobre la partida, por lo que el léxico utilizado era correcto y suficiente para expresar todas las acciones con las que estaban familiarizados en las partidas. Además, comprendieron todas las acciones que se requerían de ellos fuera más o menos elaborado el mensaje, puesto que se refería a términos, contextos y acciones con los que ya estaban familiarizados. Precisamente esto caracteriza a los niveles A2 y B1. Debido al grado de complejidad de algunas oraciones que recibieron, se puede encuadrar la comprensión en un B1.

El grado de adaptación de la producción de su discurso a la celeridad del contexto y, aun así, la consecución de su intención cuando comunican el mensaje eleva su nivel de contenido pragmático a B1. Pueden expresar el mismo mensaje de diferentes maneras, más simples y complejas, y, además, emiten un discurso coherente mostrando elementos de cohesión del texto, como el uso de deixis. La contextualización de este discurso en una partida de videojuegos hace que tengamos que prestar especial atención a este aspecto de la lengua, puesto que ofrece una explicación a que, a pesar de la incorrección gramatical, el discurso sea efectivo y comprensible.

\section{Resultados de las pruebas individuales}

Posterior a este análisis del discurso, se llevaron a cabo dos pruebas, una de la destreza de expresión oral y otra de comprensión escrita descritas en el apartado Métodos. La prueba no es preceptiva y su objetivo es orientar al participante sobre el nivel del que parte. Para que haya correlación entre los resultados de los exámenes escritos y orales, se propone también dividir las pruebas orales entre los niveles del MCERL. Para evaluarlas, se atiende a los contenidos gramaticales, léxicos, funcionales, pragmáticos, además de la pronunciación en el caso de la prueba oral, sirviendo de apoyo las rúbricas de autoevaluación que ofrece la página web oficial del MCERL.

Los resultados que a continuación se detallan son los obtenidos en cada destreza atendiendo a través de las pruebas descritas anteriormente. Se le asigna dos niveles en esta tabla a aquellos jugadores que hayan demostrado dominar un nivel y comenzar a mostrar aspectos del nivel inmediatamente superior.

Tabla 2.

Resultados de las pruebas individuales

\begin{tabular}{ccc} 
Jugador & $\begin{array}{c}\text { Resultado prueba } \\
\text { comprensión escrita }\end{array}$ & $\begin{array}{c}\text { Resultado prueba } \\
\text { expresión oral }\end{array}$ \\
\hline
\end{tabular}




\begin{tabular}{ccc}
\hline $\mathrm{J} 1$ & $\mathrm{~B} 2$ & $\mathrm{~B} 2 / \mathrm{C} 1$ \\
\hline $\mathrm{J} 2$ & $\mathrm{~B} 2 / \mathrm{C} 1$ & $\mathrm{~B} 2 / \mathrm{C} 1$ \\
\hline $\mathrm{J} 3$ & $\mathrm{~B} 1$ & $\mathrm{~B} 2$ \\
\hline $\mathrm{J} 4$ & $\mathrm{~B} 2$ & $\mathrm{~B} 2 / \mathrm{C} 1$ \\
\hline $\mathrm{J} 5$ & $\mathrm{~B} 2$ & $\mathrm{~A} 2 / \mathrm{B} 1$ \\
\hline $\mathrm{J} 6$ & $\mathrm{~B} 1$ & $\mathrm{~B} 2$ \\
\hline $\mathrm{J} 7$ & $\mathrm{~B} 1$ & $\mathrm{~B} 1 / \mathrm{B} 2$ \\
\hline $\mathrm{J} 8$ & $\mathrm{~A} 2 / \mathrm{B} 1$ & $\mathrm{~A} 2$ \\
\hline $\mathrm{J} 9$ & $\mathrm{~B} 1$ & $\mathrm{~B} 2$ \\
\hline $\mathrm{J} 10$ & $\mathrm{~A} 2$ & $\mathrm{~A} 1$ \\
\hline $\mathrm{J} 11$ & $\mathrm{~A} 2$ & $\mathrm{~A} 1$ \\
\hline $\mathrm{J} 12$ & $\mathrm{~B} 1$ & $\mathrm{~B} 1$ \\
\hline $\mathrm{J} 13$ & $\mathrm{~B} 2$ & $\mathrm{~A} 2 / \mathrm{B} 1$ \\
\hline $\mathrm{J} 14$ & $\mathrm{~B} 1$ & $\mathrm{~B} 2 / \mathrm{C} 1$ \\
\hline $\mathrm{J} 15$ & $\mathrm{~B} 2 / \mathrm{C} 1$ & $\mathrm{~B} 2$ \\
\hline $\mathrm{J} 16$ & $\mathrm{~B} 1$ & $\mathrm{C} 1$ \\
\hline $\mathrm{J} 17$ & $\mathrm{C} 1 / \mathrm{C} 2$ & $\mathrm{~B} 1$ \\
\hline $\mathrm{J} 18$ & $\mathrm{~B} 1$ & $\mathrm{~A} 2$ \\
\hline $\mathrm{J} 19$ & $\mathrm{~B} 2 / \mathrm{C} 1$ & $\mathrm{~B} 2 / \mathrm{C} 1$ \\
\hline $\mathrm{J} 20$ & $\mathrm{~B} 2 / \mathrm{C} 1$ & $\mathrm{~B} 1$ \\
\hline $\mathrm{J} 21$ & $\mathrm{~B} 2$ & $\mathrm{~B} 1$ \\
\hline $\mathrm{J} 22$ & $\mathrm{C} 1 / \mathrm{C} 2$ & $\mathrm{~B} 1$ \\
\hline $\mathrm{J} 23$ & $\mathrm{~B} 1$ & $\mathrm{~B} 1$ \\
\hline $\mathrm{J} 24$ & $\mathrm{~B} 1 / \mathrm{B} 2$ & $\mathrm{~A} 1$ \\
\hline $\mathrm{J} 25$ & $\mathrm{~A} 2$ & $\mathrm{~B} 2$ \\
\hline $\mathrm{J} 26$ & $\mathrm{~B} 2$ & $\mathrm{~B} 1 / \mathrm{B} 2$ \\
\hline $\mathrm{J} 27$ & $\mathrm{~A} 2 / \mathrm{B} 1$ & $\mathrm{~B} 2$ \\
\hline $\mathrm{J} 28$ & $\mathrm{~B} 2 / \mathrm{C} 1$ & $\mathrm{~B} 2$ \\
\hline $\mathrm{O} 1$ & $\mathrm{~B} 2$ & \\
\hline $\mathrm{O} 2$ & $\mathrm{~B} 2$ & \\
\hline & & \\
\hline
\end{tabular}

No existe una correlación entre los resultados de las destrezas escrita y oral. Tal y como confirman los jugadores a través de las entrevistas, esto se debe a la destreza que más han puesto en práctica. Es decir, aquellos jugadores que tienen mejor nivel escrito apuntan que normalmente se expresan de manera escrita y no de manera oral y viceversa. Este dato es coincidente en todos los jugadores en la misma circunstancia.

Dada la peculiaridad del contexto informal que permiten los eSports, es difícil, si no imposible, categorizar en niveles estancos el conocimiento de la lengua del alumnado, que se adapta y se desarrolla constantemente dependiendo del contexto, por lo que es conveniente, como profesionales docentes que ahondemos en los conocimientos que muestra nuestro alumnado, ya que los niveles del MCERL no se deben tratar como un fin, sino como un medio, como ya se exponía en este documento cuando se publicó por primera vez (Ministerio de Educación, Cultura y Deporte, 2002): «hay que dejar claro desde el principio que NO nos proponemos decir a los profesionales lo que tienen que hacer o de qué forma hacerlo. Nosotros planteamos preguntas, no las contestamos». 


\section{La dimensión comunicativa en los resultados}

La particularidad de este estudio es fundamentalmente el entorno en el que desarrolla, que es el de los videojuegos a nivel competitivo. Atendiendo a esta característica clave, no podemos, entonces, dejarnos atrás las dimensiones que se ponen en juego en cualquier intercambio comunicativo, especialmente porque estamos entrando en contacto, no solo con un código nuevo, sino con un modo diferente de entender el mundo (Centro Virtual Cervantes, 2018). En este sentido, el contenido del mensaje no puede ser en exclusiva el foco del estudio, sino que hay que entenderlo como parte del medio. Así, el proceso comunicativo específico en los eSports no cobra interés solamente por su contenido, sino por las características del medio en sí. Hay que entender las características del medio y el medio en sí mismo, ya que este forma parte del mensaje (Fernández Navas, 2015; McLuhan, 1962, 2001).

En este análisis nos vamos a centrar en las dos dimensiones principales que toman presencia en este aprendizaje, a partir de las cuáles se desarrollan las destrezas características de cada nivel que habíamos indicado con anterioridad. Estas son el uso social de la lengua y la pluriculturalidad. De acuerdo con esto, el aprendiz podría tomar dos perspectivas (Centro Virtual Cervantes, 2018): como agente social, en la que se le acredita un conocimiento de los elementos que constituyen el sistema de la lengua y la capacidad de desenvolverse en las situaciones habituales de comunicación que se dan en la interacción social; y como hablante intercultural, en la que se le atribuye la capacidad de identificar los aspectos relevantes de la nueva cultura a la que accede a través de la lengua extranjera y de establecer puentes entre la cultura de origen y la de los países que hablan esta lengua.

Durante las entrevistas aparecen de diversas maneras estas dimensiones, ya que la primera explica las diferentes estrategias que ponen en juego los aprendices para comunicarse, y el desarrollo de la segunda facilita la comunicación, al tratarse de un juego online en el que tienen que comunicarse con personas de diferentes culturas. Desarrollar estas dimensiones son fundamentales para poder contribuir al equipo, compuesto de a menudo de jugadores de diferentes orígenes, y así conseguir la victoria deseada en la competición. Cuando uno de los jugadores detectó la relación que unía a un grupo determinado de jugadores, decidió cambiar su aprendizaje del inglés, idioma en el que se jugaba, por el turco.

[...] hasta que se llenó el servidor de turcos y tocó aprender un poquito de turco. Hay que buscarse la vida. De turco a turco te lo vende más barato, pues entonces tú te haces pasar por turco (extracto de entrevista con J6).

Un 13,33\% de los jugadores han demostrado un nivel básico, (A1 y A2), en estas dimensiones. Es decir, en su perspectiva como agente social, cuentan con un repertorio limitado de recursos lingüísticos sencillos, como estructuras sintácticas básicas, en las que se incluyen frases o fórmulas memorizadas); en compensación a sus dificultades de comunicación, recurren a sus destrezas, habilidades y actitudes, por ejemplo, las de imitación de otros jugadores o la capacidad de preguntar por aquello que no entienden; $\mathrm{y}$, aunque resulte evidente su acento extranjero $\mathrm{y}$, a veces, esto de lugar a interrupciones o malentendidos, por lo general, la comunicación es comprensible y clara, entre otras características.

En la dimensión cultural, observamos diversas actitudes de menor a mayor profundidad, típicas de este nivel. Cuanto más tiempo haya estado expuesto a la diversidad cultural y, por tanto, más haya practicado sus destrezas lingüísticas, más consolidada estará su perspectiva como hablante intercultural. Así, el jugador empieza a 
tomar conciencia de la diversidad cultural y de la influencia que puede tener la propia identidad cultural en la percepción e interpretación de otras culturas, hasta, finalmente aprovechar la diversidad cultural como una fuente de enriquecimiento de la propia competencia intercultural.

Un 30\% ha demostrado un nivel B1 desde la perspectiva de agente social y un $43,33 \%$ un B2. Es decir, un 30\% de los jugadores cuenta con un repertorio amplio pero sencillo de vocabulario, estructuras y fórmulas aprendidas, en contraste con aquellos que se encuentran en un nivel B2, que disponen de los recursos lingüísticos necesarios para comunicarse con un grado de fluidez, precisión y naturalidad que implica que sus interlocutores no tengan que hacer un esfuerzo especial. Con el objetivo de suplir esta falta de habilidad lingüística, el aprendiz que demuestra un nivel B1 en esta dimensión, pondrá en juego los conocimientos generales de la cultura de la lengua en la que se está comunicando (referentes culturales, normas y convenciones de carácter sociocultural) para desenvolverse en las diferentes situaciones comunicativas en las que participan. El nivel de conciencia de la lengua que un aprendiz en nivel B2 tiene le permite evitar errores que den lugar a malentendidos y le hace capaz de utilizar suficientes recursos como para salvar situaciones de ambiguiedad y aclarar lo que el interlocutor ha querido decir. La comunicación en un nivel B1 será en un registro neutro, aunque con suficiente flexibilidad como para adaptarse a diferentes situaciones. Aunque vacilen o hagan pausas para pensar lo que van a decir y cometan errores (especialmente de pronunciación), se expresan con razonable corrección. Además, en caso de que sea necesario, saben cómo pedir a alguien que aclare o desarrolle lo que acaba de decir. Estas habilidades se desarrollan en el nivel B2, donde poseen un repertorio lingüístico amplio, suficiente para expresarse con argumentos y matices, sin errores importantes de formulación y con una pronunciación clara. Sumada a las habilidades del B1, en el nivel B2 también son capaces de considerar el efecto que producen sus comentarios.

Al principio nada más que [sabes] decir "vamos por este lado o este otro", "hay un enemigo por ahí o por allá" y cuando ya ves que te están zurrando porque no puedes comunicarle a tu amigo qué es lo que te está pasando realmente como a ti te gustaría en español, pues ya poco a poco tú vas escuchando a ellos cómo sí que lo hacen, te quedas con sus palabras, se te quedan a ti también, las vas usando y poco a poco las vas aprendiendo. Entre eso y foros y tal y cual, al final uno aprende (extracto de la entrevista con J14).

En la dimensión cultural, como ocurría en el nivel básico, observamos diversas actitudes de menor a mayor profundidad, típicas de este nivel intermedio. Además, hablaríamos de una dimensión cultural de nivel intermedio de la lengua que implica la no separación de los niveles B1 y B2. Así, encontramos que un alto porcentaje de los jugadores, que sí son capaces de identificar, analizar y aprovechar el enriquecimiento cultural que se derivan de la interacción de distintos sistemas de valores, comportamientos y percepciones de la realidad, propios de cada cultura. Existe un fortalecimiento de las motivaciones, la sensibilidad y la apertura hacia otras culturas, que le lleva a alcanzar un nivel de información básico de los aspectos culturales, en especial aquellos más generales y conocidos, como sus principales características geográficas, demográficas, económicas, etc., de aquellos países con los que tiene contacto.

Un 13,33\% de los jugadores demostraron un nivel $\mathrm{C} 1$ en estas dimensiones que, como agentes sociales, disponen de un repertorio de recursos lingüísticos lo suficientemente amplio y rico como para comunicarse con fluidez y naturalidad, casi sin 
esfuerzo; son capaces de expresar matices de significado eliminando ambigüedades y confusiones; su comunicación es fluida y espontánea, con pocas vacilaciones y poseen la capacidad de adecuar su registro a cada tipo de situación.

En la dimensión cultural, se caracteriza especialmente por tomar la iniciativa en la búsqueda, incorporación y activación estratégica de nuevos conocimientos culturales, socioculturales y lingüísticos, destrezas y actitudes para una mayor comprensión de la nueva realidad desde una perspectiva plurilingüe y pluricultural.

Representamos en forma de gráfica (Figura, 1) los porcentajes de jugadores de acuerdo con sus dimensiones comunicativas:

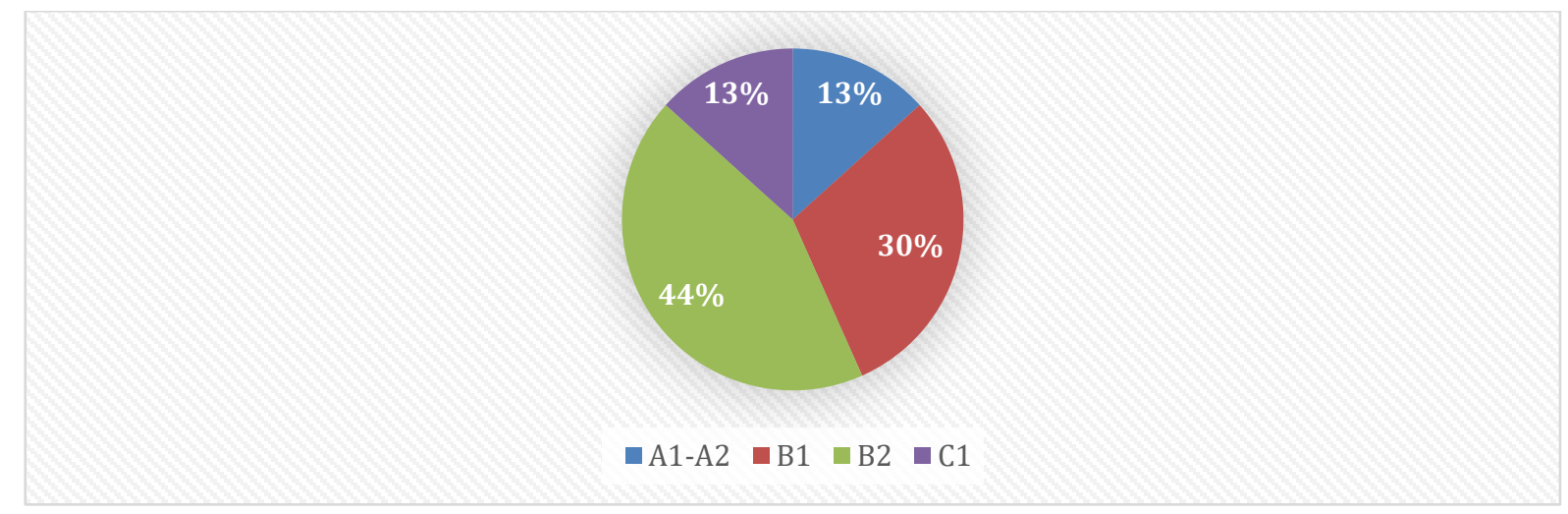

Figura 1. Porcentaje de jugadores de acuerdo con su nivel de uso social y pluriculturalidad

Los resultados en las dimensiones como agente social y pluricultural se corresponden siempre al nivel más alto de destreza que han demostrado en las pruebas. Esto se debe a que, aunque no hayan practicado una destreza en concreto, sí que ha desarrollado estas dimensiones acordes a su nivel de conocimiento global de la lengua, ya que la competencia cultural se desarrolla en el uso de la misma (Miquel, 2004).

\section{Conclusiones}

\section{El potencial para aprender una segunda lengua extranjera con videojuegos}

El análisis de estos resultados nos ayuda a tener una idea más profunda del conocimiento que se genera de una lengua extranjera cuando se juega a videojuegos a nivel competitivo. Este conocimiento puede ayudarnos a comprender mejor una realidad que están viviendo cada vez más jóvenes, que entran en contacto con otra lengua y cultura de manera informal. Los autores de este artículo creemos que estos resultados pueden ayudar a algunos docentes que se encuentran a estos jugadores en el aula a aprovechar este conocimiento que se genera. Lejos de pretender la generalización de este conocimiento, nuestra investigación, al encuadrarse en una metodología cualitativa, busca transferibilidad (Korstjens y Moser, 2017; Lincoln y Guba, 1985; Maxwell, 2010, 2012; Tracy, 2010), es decir que fruto de la comprensión de procesos y realidad complejas, puedan encontrarse cuestiones susceptibles de ser implantadas en contextos diferentes por otro investigador (Flick, 2018). 
Tras el análisis de los audios y vídeos de las partidas en semifinal y la observación de las partidas entre jugadores, se puede deducir que existe una jerga en el mundo de los videojuegos y, es más, que cada videojuego tiene su propia jerga y para comunicarse con éxito en él es necesario conocerla. El punto en común que podemos encontrar es el uso de términos, es decir, aquellas palabras que tienen un significado preciso en un contexto determinado. Encontraríamos tres tipos principales: el préstamo, es decir, cuando se utiliza la palabra del inglés directamente, por ejemplo, cuando los jugadores usan el término noob para referirse a los novatos o a los comportamientos típicos de los novatos; los préstamos que ya han iniciado el proceso de incorporación gráfica y fonética al español, como pushear; y el calco, utiliza una expresión que existe en español, pero en un contexto o con un valor que corresponde al uso inglés, como es empujar, para referirse a una estrategia determinada, la acción push en el juego.

Aunque el análisis linguiístico de estos términos podría desarrollarse en otras investigaciones, lo que nos debe interesar es en qué afecta al aprendizaje estos. En primer lugar, se comienzan a construir significados, aunque no se reconozca o comprenda el significante de manera aislada. En un extracto de la entrevista con O2 encontramos la siguiente afirmación que nos ayudará a entender la importancia de los términos del juego en el aprendizaje de una lengua: «[cuando empecé a jugar] yo no sabía qué significaba start, pero cuando pulsaba empezaba el juego y claro, lo entendía». Si se reflexiona sobre esta exposición al término y se toma control sobre ello, puede derivar en un aumento del contenido léxico y gramatical de la lengua en la que está teniendo lugar la acción, en este caso, inglés y podríamos empezar a hablar de aprendizaje de la lengua.

Este concepto de inmersión lingüística en el que se utiliza el idioma como un medio para alcanzar un fin es el que se propone en el sistema AICLE (Casal \& Moore, 2009; Lorenzo, Casal \& Moore, 2009; Navarro Pablo \& García Jiménez, 2018; Nieto, 2016). Los videojuegos permiten esta inmersión de una manera más rápida y fácil, obteniendo los mismos resultados que si viajáramos a otro país, ya que es muy influyente en el aprendizaje la exposición y el uso que tienen los jugadores al idioma (González \& Egido, 2017), además de en el desarrollo del perfil como hablante intercultural del jugador/aprendiz.

Vemos entonces que una de las claves de este aprendizaje es que el conocimiento se construye a través de la interacción, en la que el jugador desarrolla su dimensión como agente social. Por un lado, se desarrollan las habilidades comunicativas y se adquieren las estructuras gramaticales gracias a la exposición a un input comprensible (Krashen, 1985). Por otro lado, el output invita al aprendiz a reflexionar sobre sus conocimientos de la lengua extranjera a través de la puesta en práctica de estos. Los videojuegos componen un espacio favorable para aprender a situar y construir significados a través de la experiencia (Gee, 2004).

Además, los jugadores con un nivel B2 o superior afirman haber asistido a clases extraescolares de la lengua extranjera o han comenzado a estudiar la lengua de manera autónoma a través de manuales o guías en internet, por lo que no solo su exposición a la lengua extranjera en el videojuego ha contribuido a su desarrollo de las destrezas, sino que también ha sido necesario un estudio controlado de esta.

[Te entran más ganas de aprender inglés] porque frustra bastante no saber lo que están diciendo o incluso, no sólo eso, me refiero, cualquier tipo de vídeo o lo que sea prefiero verlo en inglés y hay muchas cosas que me entiendo y eso hace que decía veces, "mira, voy a intentar aprender inglés o 
voy a intentar entenderlo como buenamente pueda y ya está" (extracto de la entrevista con J15).

Igualmente, no parece existir una influencia del número de horas que se dedica a jugar al videojuego, pero sí qué tipo de exposición se tienen con respecto a la lengua extranjera. Para esto va a ser determinante la elección del videojuego: cuanto más permita hablar e interactuar entre los jugadores, más necesidad habrá de utilizar diversas funciones de la lengua y más exposición y producción de gramática, léxico y recursos pragmáticos serán necesarios para cumplir estas funciones. Se señalaba en algunas entrevistas que el salto a multijugador y a multijugador online no ha implicado siempre una mejora de la comunicación entre los jugadores, ya que hay juegos que se prestan más a una conversación entre jugadores, como los $\mathrm{MMORPG}^{1}$, en los que aparecen temas no relacionados con el juego, ya que las conversaciones son más largas y las relaciones interpersonales se pueden desarrollar más; y juegos en los que las conversaciones, la temática y el vocabulario son más restringidos, como los MOBA ${ }^{2}$. Para el desarrollo del aprendizaje de la lengua en este último tipo de juegos son claves los metatextos, es decir, los textos que se producen sobre el juego externos al videojuego en sí, como entrevistas o noticias. A menudo, estos textos se encuentran en la segunda lengua extranjera y conforman una parte importante en la práctica de la lengua extranjera por la motivación de aprender más sobre el videojuego. En los videojuegos a nivel competitivo cobran especial importancia los metatextos, para estudiar estrategias nuevas y cuanto mayor es el nivel al que se compita en los torneos, más posibilidad habrá de jugar en un equipo internacional. Estas circunstancias implican una mayor relevancia en el aprendizaje de la lengua extrajera.

De acuerdo con los resultados obtenidos, por tanto, implican que, cuanto mayor es la necesidad de comunicarse y mayor es la exposición del input y la producción en la lengua extranjera, más posibilidades habrá de desarrollar esta lengua (Barrios Espinosa y García Mata, 2005; Long, 1983). Además, los eSports ofrecen una plataforma en la que crear una comunidad intercultural, que desarrolla esta conciencia, pero que además anima al jugador a tener en cuenta otros aspectos culturales y encontrar recursos lingüísticos para la comunicación con los otros posibles miembros del grupo. En posteriores investigaciones será interesante ahondar en los mecanismos utilizados por los jugadores para aprenderlas, de manera que nos centremos en el cómo además de en el qué, como se ha querido plasmar en este estudio.

\section{Referencias}

Alcaraz, N. (2015). Evaluación versus calificación. Aula de encuentro, 2(17), 209-236.

Barrios Espinosa, E., y García Mata, J. (2005). Teaching and learning foreign languages. En TEFL in Secondary School (Vol. 32, pp. 115-133). Universidad de Granada.

Begg, M., Dewhurst, D., y Macleod, H. (2005). Game-Informed Learning: Applying Computer Game Processes to Higher Education. Innovate: Journal of Online Education, 1(6).

\footnotetext{
${ }^{1}$ Sigla en inglés de massively multiplayer online role-playing game. En español, videojuegos de rol multijugador masivos en línea.

2 Sigla en inglés de multiplayer online battle arena. En español, arena de combate multijugador en línea.
} 
Casal, S., y Moore, P. (2009). The Andalusian bilingual sections scheme: Evaluation and con-sultancy. International CLIL Research Journal, (1), 36-46.

Centro Virtual Cervantes. (2018). Plan curricular del Instituto Cervantes. Recuperado de https://cvc.cervantes.es/ensenanza/biblioteca_ele/plan_curricular/default.htm

Chen, D. (2014). Gamer perception of language learning and L2 interaction in MMORPGs. University of Birnmigham.

Chik, A. (2014). Digital Gaming and Language Learning: Autonomy and Community. Language Learning \& Technology, 2(18), 85-100.

Educo. (2017). ¿Qué son los eSports? Recuperado 8 de enero de 2019, de http://blog.educo.org/que-son-los-eSports/

EnglishProfile. (2011). Introducing the CEFR for English. Lifelong Learning Programme.

Fernández, M. V. (2006). Las TIC en la enseñanza del Inglés como Lengua Extranjera (ILE): una herramienta online y una off-line. Revista Latinoamericana de Tecnología Educativa, 2(5), 409-416.

Fernández Navas, M. (2015). Internet, organización en red y educ@ación: estudio de un caso de buenas prácticas en enseñanza superior. Universidad de Málaga.

Figueras Bates, C. (2014). Pragmática de la puntuación y nuevas tecnologías. Normas, 4, 135-160. doi: 10.7203/Normas.4.4691

Flick, U. (2007). Introducción a la investigación cualitativa (Segunda). Madrid: Morata.

Flick, U. (2018). The Concepts of Qualitative Data: Challenges in Neoliberal Times for Qualitative Inquiry. Qualitative Inquiry. doi: 10.1177/1077800418809132

Flyvbjerg, B. (2006). Five Misunderstandings About Case-Study Research. Qualitative Inquiry, 2(12), 219-245. doi: 10.1177/1077800405284363

Gee, J. (2004). Learning by design: Games as learning machines. Interactive Educational Multimedia, (8), 15-23.

Gee, J. (2007). What Video Games Have to Teach Us About Learning and Literacy. New York: Palgrave Macmillan.

Gee, J.P., y Hayes, E.R. (2010). Public pedagogy through video games: Design, resources \& affinity spaces. En J. A. Sandlin, B.D. Schultz, y J. Burdick, Public Pedagogy: Education and Learning beyond Schooling (pp. 185-193). New York: Routledge.

Gibbs, G. (2012). El análisis de datos cualitativos en investigación cualitativa. Morata.

Godwin-Jones, R. (2014). Games in Language Learning: Opportunities and Challenges. Language Learning \& Technology, 2(18), 9-19.

González, M., y Egido, I. (2017). Factores explicativos del aprendizaje de la comprensión oral en lengua inglesa en educación secundaria: comparación entre España y Holanda. Revista complutense de educación, 28(2), 591-607. doi: 10.5209/rev_rced.2017.v28.n2.49634

Graham S.L., y Hardaker C. (2017). (Im)politeness in Digital Communication. En J. Culpeper, M. Haugh, y D. Kádár, The Palgrave Handbook of Linguistic (Im)politeness. London: Palgrave Macmillan. 
Korstjens, I., y Moser, A. (2017). European Journal of General Practice, 1(24), 120 124. doi: 10.1080/13814788.2017.1375092

Krashen, S. D. (1981). Second Language Acquisition and Second Language Learning. Oxford: Pergamon.

Krashen, S.D. (1985). The Input Hypothesis: Issues and Implications,. Nueva York: Longman.

Lincoln, Y.S., y Guba, E. G. (1985). Naturalistic inquiry. California: Sage Publications.

Long, M. H. (1983). Does Second Language Instruction Make a Difference? A Review of Research. TESOL, 17(3), 359-382.

Lorenzo, F., Casal, S., y Moore, P. (2009). The effects of content and language integrated learning in European education: Key findings from the Andalusian bilingual sections evaluation project. Applied Linguistics, (3), 418-442. doi: 10.1093/applin/amp041

Martín Díaz, V. (2012). El ayer y hoy de los videojuegos y juegos digitales. En Los videojuegos y los juegos digitales como materiales educativos (pp. 19-34). Madrid: Síntesis.

Maxwell, J. A. (2010). Using Numbers in Qualitative Research. Qualitative Inquiry, 6(16), 475-482. doi: 10.1177/1077800410364740

Maxwell, J. A. (2012). Qualitative Research Design. An Interactive Approach. London: Sage.

McLuhan, M. (1962). The Gutenberg Galaxy. Toronto: University of Toronto Press.

McLuhan, M. (2001). Understanding Media: The extensions of man. Abingdon: Routledge.

Miles, M. B., y Huberman, A. M. (1994). Qualitative data analysis: An expanded sourcebook (2nd ed.). Thousand Oaks, CA: Sage.

Ministerio de Educación, Cultura y Deporte. (2002). Marco Común Europeo de Referencia para las Lenguas: Aprendizaje, Enseñanza, Evaluación (Instituto Cervantes para la traducción en españo, Trad.).

Miquel, L. (2004). Lengua y cultura desde una perspectiva pragmática. RedELE, (2), 137-161.

Morse, J. M. (1998). Designing Funded Qualitative Research. En N. Denzin y Y. S. Lincoln, Strategies of Qualitative Research (pp. 56-85). Londres: Sage.

Navarro Pablo, M., y García Jiménez, E. (2018). Are CLIL Students More Motivated? An Analysis of Affective Factors and their Relation to Language Attainment. Porta Linguarum, (29), 71-90.

Nieto, E. (2016). The impact of CLIL on the acquisition of the learning to learn competence in secondary school education in the bilingual programmes of Castilla-La Mancha. Porta Linguarum, (2), 21-34.

Pérez, Á. I., Soto, E., Sola, M., y Serván, M. J. (2009). Aprender Cómo aprender. Autonomía y responsabilidad: el aprendizaje de los estudiantes. Madrid: Ediciones Akal.

Pérez Gómez, A. I. (2000). La cultura escolar en la sociedad neoliberal. Madrid: Morata. 
Pérez Gómez, A. I. (2004). La cultura escolar en la sociedad neoliberal. Madrid: Morata.

Pérez Gómez, A. I. (2012). Educarse en la era digital. Madrid: Morata.

Pindado, J. (2005). Las posibilidades educativas de los videojuegos. Una revisión de los estudios más significativos. Pixel-Bit. Revista de Medios y Educación, (26), 5567.

Pozo, I. (2016). Aprender en tiempos revueltos. Madrid: Alianza Editorial.

Reinders, H. (2014). Can I Say Something? The Effects of Digital Gameplay on Willingness to Communicate. Language Learning \& Technology, 2(18), 101123.

Reinhardt, J., y Sykes, J. (2014). Digital game and play activity in L2 Teaching and Learning. Language Learning \& Technology, 18(2), 2-8.

Reinhardt, J., y Sykes, J.M. (2014). Special Issue Commentary: Digital Game Activity in L2 Teaching and Learning. Language Learning \& Technology, 2(18), 2-8.

Ridao Rodrigo, S., y Rodríguez Muñoz, F. J. (2013). Problemas de puntuación en contextos digitales: análisis de comentariso en foros de youtube. Anuario de EStudios Filológicos, 36, 83-105.

Sampedro Requena, B, E. (2012). La perspectiva psicológica del videojuego en la infancia y la adolescencia. En M. D. V., Los videojuegos y los juegos digitales como materiales educativos (pp. 35-59). Madrid: Síntesis.

Santos Guerra, M. A. (2005). Hacer visible lo cotidiano. Teoría y práctica de la evaluación cualitativa de los centros escolares. Madrid: Akal.

Simons, H. (2011). El estudio de caso: teoría y práctica. Madrid: Morata.

Stake, R. E. (2010). Investigación con estudio de casos. Madrid: Morata.

Sundqvist, P., y Sylvén, L. K. (2012). World of VocCraft: Computer Games and Swedish Learners' L2 English Vocabulary. En H. Reinders (Ed.), Digital Games in Language Learning and Teaching (pp. 189-208). Recuperado de doi: 10.1057/9781137005267_10

Sylvén, L. K., y Sundqvist, P. (2012). Similarities between playing World of Warcraft and CLIL. Apples-Journal of Applied Language Studies, 2(6), 113-130.

Sylvén, L. K., y Sundqvist, P. (2015). Extramural English in relation to CLIL: Focus on young language learners in Sweden. En S. Marsh, M. L. Pérez Cañado, y J. Ráez Padilla (Eds.), CLIL in Action: Voices from the classroom. (pp. 47-62). Newcastle-upon-Tyne: Cambridge Scholars Publishing.

Tracy, S. J. (2010). Qualitative Quality: Eight "Big-Tent" Criteria for Excellent Qualitative Research. Qualitative Inquiry, 10(16), 837-851. doi: $10.1177 / 1077800410383121$

Whymaps. (2017). \#WHYGAMES: Assassin's Creed Origins \#jugareslaostia. Recuperado de https://www.youtube.com/watch?v=MfmJnX5PDVU

Fecha de recepción: 10/01/2019

Fecha de revisión: 22/05/2019

Fecha de aceptación: 15/07/2019 